\title{
Measurement of retinal function with flash electroretinography in Chinese patients with hyperlipidemia
}

\author{
Fatih C. Gundogan • Abdullah Ilhan • Umit Yolcu • \\ Salih Altun
}

Received: 14 October 2014 / Accepted: 30 October 2014 /Published online: 15 November 2014

(C) Springer-Verlag Berlin Heidelberg 2014

Dear Editor,

We congratulate Zhang et al. for their study evaluating the retinal functional changes in hyperlipidemia [1]. The authors reported significant changes in full-field electroretinograms (ffERG) in patients compared to control healthy subjects. The study included 626 patients with hyperlipidemia. This high number of patients alone makes the study valuable. The results carry significant consequences that should be discussed.

The authors reported significant reductions in the a- and bwave amplitudes in rod response, maximum rod-cone response, oscillatory potentials, cone responses, and $30-\mathrm{Hz}$ flicker responses. Similarly, they reported significantly longer implicit times. The authors found these significant differences persisted even after adjusting for age and gender. Total amplitudes of OPs were $40 \%$ lower, and implicit time was $15 \%$ longer in hyperlipidemic patients. We want to emphasize that lens sclerosis may be a confounding factor in the results. In a recent report including 11,591 participants, hypercholesterolemia was found to be an independent risk factor in the development of cataracts [2]. In that study, other independent risk factors were lower monthly household income, lower education, hypertension, and diabetes mellitus. FfERG is a mass retinal response to a flash of light. Cataracts or lens opacities reduce the intensity of the light reaching the retina that in turn

F. C. Gundogan $(\bowtie) \cdot$ S. Altun

GATA Medical School, Ophthalmology, GATA Göz Hst. AD Bşk.

Etlik, Keçiören, 06010 Ankara, Turkey

e-mail: fgundogan@yahoo.com

\author{
A. Ilhan \\ Erzurum Military Hospital, Ophthalmology Service, \\ Erzurum, Turkey \\ U. Yolcu \\ Sarikamis Military Hospital, Ophthalmology Service, Kars, Turkey
}

reduces the response of the retina. We want to ask to the authors whether they collected data about the lens opacity in the patients. It would be better to take the lens opacity classification system (LOCS) into consideration in the study and adjust the statistical analyses for the LOCS.

Conflict of interest All authors certify that they have no affiliations with or involvement in any organization or entity with any financial interest (such as honoraria; educational grants; participation in speakers' bureaus; membership, employment, consultancies, stock ownership, or other equity interest; and expert testimony or patent-licensing arrangements), or non-financial interest (such as personal or professional relationships, affiliations, knowledge, or beliefs) in the subject matter or materials discussed in this manuscript.

\section{References}

1. Zhang B, Qiu Q, Yin L, Yao Y, Wang C, Wu X (2014) Measurement of retinal function with flash-electroretinography in Chinese patients with hyperlipidemia. Graefes Arch Clin Exp Ophthalmol 252:1385-1392. doi:10.1007/s00417-014-2726-Z

2. Rim TH, Kim MH, Kim WC, Kim TI, Kim EK (2014) Cataract subtype risk factors identified from the Korea National Health and Nutrition Examination survey 2008-2010. BMC Ophthalmol 14:4. doi:10.1186/1471-2415-14-4 